\title{
Study on the Importance of "Jumping" in Chinese Classical Dance
}

\author{
Yanchi Zhou ${ }^{1, *}$ \\ ${ }^{1}$ School of Music and Performing Arts, Mianyang Teachers' College, Mianyang, Sichuan 621000, China \\ *Corresponding author. Email: 1046290187@qq.com
}

\begin{abstract}
In the process of dance performances, jumping movements can accurately express the emotional routes of the characters in dance works such as happiness, anger, sorrow and joy; jumping movements better show the charm of dance and create a better viewing experience for the audience; the degree of beauty of the jumping action has a great influence on the stage effect presented by the dance work, and the jumping is of great significance to shaping a perfect stage image. Only when dancers actively carry out jumping training, flexibly use their hands and eyes together, and skillfully use jumping movements, can they present better stage effects to the audience.
\end{abstract}

Keywords: Jumping, Classical dance, Importance, Jumping training, Stage performance.

\section{INTRODUCTION}

As a form of artistic expression that uses body movements to express emotions, dance must have certain requirements for certain joints, ligaments and muscles of the body when completing certain movements. It plays an extremely important role in completing a single action, various combinations, and dance repertoire. Jump training is necessary to improve the quality of dance movements. Jump training is also essential for improving dance skills. Therefore, in order to improve the jumping ability of the body, it is a must to first strengthen the emphasis on jumping ability. Only with good jumping training to improve the improvement of muscle ability can a solid foundation be laid for the stability, expressiveness and control of dancers in performance, so as to make the dancers have the ability to complete good works. Secondly, in dance performances, jumping is one of the most important forms of dance. In the training process of jumping movements, it is necessary to pay attention to a certain degree of science and regularity.

\section{CLASSIFICATION OF CLASSICAL DANCE SKILLS}

\subsection{Jete}

The jete is an important part of the basic training in Chinese classical dance. The jete mainly refers to the small jump motions performed by the dancers during the performance. The movements of the jete are simple, so that beginners can know how to rise and fall, and perform jete training. One part of the action is to train the dancer's jumping foundation, and the other part is for the dancer to perform stage movements.

\subsection{Mid Jete}

Compared with the jete, the mid jete is relatively larger in the amplitude and strength of the jump. Mid jete training is an important means to increase the elasticity of jumping [1]. The most significant impact of mid jete training is to improve the control of the leg muscles. Knee exercises through mid jete can speed up the flexibility of the calf and under the leg, and increase the explosive power of the leg muscles and the toughness of the knee. It can also make quick transitions between action connections, which is very helpful for action transitions in fast rhythms. 


\subsection{Big Jete}

The big jete mainly refers to the large-scale expansion of the dancer in the air and the maximum occupation of space height. To be precise, a big jete refers to a jumping action where the dancer expands to a large extent while maintaining an accurate dance posture while having an obvious movement track [2]. The big jete is to train the aerial technical ability, but also to improve the performance of the dancers' limbs. The stage performance of the big jump requires high technical ability of the dancers. The dancer's big jete is to show the dance moves.

\section{THE IMPORTANCE OF JUMPING IN CHINESE CLASSICAL DANCE}

\subsection{The Importance of Jumping in the Training Process of Chinese Classical Dance}

The dancers show the charm of dance through jumping movements, which brings a great viewing experience to the audience. The beauty of the dancers' jumping movements has a great influence on the stage effect presented by the dance works. The exercise of the dancer's own jumping action is very effective in shaping a perfect stage image. Many dancers present classic stage performances to the audience, which is inseparable from the dancer's usual jumping training.

In the usual jumping training, the muscles of the abdomen and buttocks of the body can be greatly exercised through jumping training. Accurate jumping movements can effectively exercise the muscle groups of various parts of the dancer's body, including the small muscle groups on the abdomen and the muscles of the buttocks. When performing dance training, the muscles of the legs are actively stretched, and the muscles of the abdomen are actively stretched so that the muscles of the legs and abdomen can be trained at the same time. Then through jump training, the strength of the leg muscles can be exercised to ensure the stability of the dancer's body and the sense of neat movement. It can also train the muscles of the abdomen and legs of the dancers so that each jump action of the dancers can be completed with more texture, and it can also enhance the muscle line sense of the dancers' whole body. When dancers are performing jump training, if they want to jump high and have a certain specific performance time in the air, they need to strengthen the ability of the leg muscles. In the process of jumping, the leg strength can be actively tightened, giving the dancer a stronger jumping power, and enhancing the sense of flight can provide time for the dancer to perform in the air. In the process of exercise, it is necessary to strengthen the exercise of the muscles on the one hand, and on the other hand to maintain the state of relaxation and relaxation of the muscles to avoid accidents of muscle strains. Only when the sense of connection between the movements is perfect can the jump be higher and more stable. For example, there is a connection of two consecutive jumping movements. When performing a performance, it is difficult for a dancer to show flexibility and coherence if the knee joint has not been trained to a certain extent.

A professional dancer not only has strict requirements on the dancer's strength, movement stability, physical coordination and flexibility in jumping expression. The strength refers to the strength of the dancer's performance, mainly by controlling the bounce ability of the muscles and the tension of the dance movements. Action stability specifically refers to the flexibility of the dancer's joint movement. When an actor is performing, the dancer's actions must be coherent, fluid, graceful, and not rigid. The control of dance movements comes to a large extent from the actor's own muscle strength support, the control of movement balance, and the control of the strength of dance movements. The perfect display of all this is inseparable from the dancer's usual jumping practice.

\subsection{The Importance of Jumping in the Teaching Process of Chinese Classical Dance}

In the dance classroom teaching, the author has a deep understanding of the practice of jumping, and has rich teaching experience. As a dance substitute teacher, the training of jumping ability cannot be ignored or underestimated, because the training of ability is not only to prepare for jumping, but also very important in all kinds of skills in dance. A professional dancer can control his body freely after good jumping training. It is not only good for movement stability, body coordination and flexibility of dance expression, but also good for the coordination of various muscle tissues of the body. Regardless of the dancer's ability to change movements and dance movements, for dancers, there will be multiple rapid changes in their performances. This is related to a body with good 
muscle ability. The exercise of a good physical fitness has a great relationship with the jump training for students in normal teaching. A good jumping training system is also very important, because in this way can the dancer's muscle ability and physical fitness be accurately exercised, which is not only helpful for jumping skills, but also for all technical skills. Jumping exercises in the teaching process can make the dancer's physical fitness fully developed, and the dancer's dexterity, flexibility and responsiveness can be greatly improved.

\section{THE IMPORTANCE OF "JUMPING" IN CHINESE CLASSICAL DANCE}

\subsection{The Importance of "Jumping" in Chinese Classical Dance "Kong Yiji"}

Art is the reflection of real social life through images. These vivid and touching artistic images can subtly influence the emotions of the audiences and make the works produce special effects. In dance performances, the use of jumping can create rich imagination and emotion, and better create the artistic conception of the work. Dance works convey profound meaning and spirit to complete a work is very important. Jumping can convey the "god" of dance, to create a perfect dance artistic conception. In "Kong Yiji", in the dance movement in which Kong Yiji appeared, the dancers performed a series of jumping movements. These jumping movements are lively and lively, showing the lively appearance of Kong Yiji, interesting and lively, showing the atmosphere of Kong Yiji's appearance, and perfectly shaping the appearance of the dance character Kong Yiji. At this time, the atmosphere of the whole dance is playful, relaxed and humorous, which paved the way for Kong Yiji's sad ending at the end of the dance. In the first stage of the dance "Kong Yiji", the background behind comes from the famous composer Lin Hai's "Pipa Xing". At this time, the whole dance is divided into playful and lively. Driven by the music, the dancers presented the lively and lovely image of Kong Yiji in front of the audience through a series of jumping movements and the music, bringing the audience into a relaxed, humorous and interesting dance atmosphere. In the climax of the dance "Kong Yiji", it shows the scene that Kong Yiji was drunk, stealing books and being beaten. At this time, the dancers brought the whole stage atmosphere to a climax through a series of mid jetes and big jetes.
The dancer's faster and faster jumping movements vividly show Kong Yiji's nervousness when being beaten, nowhere to dodge, and a strong sense of rhythm. In this performance, the classical dance "Kong Yiji" broke the audience's solid thinking about classical dance in the past. In the past audience's impression, most classical dances are exquisite body movements, beautiful body language, and have a high degree of appreciation. However, in the second part of "Kong Yiji", the dancer's movements are exaggerated and humorous, relaxed and humorous, breaking the traditional classical dance action form and showing Kong Yiji's down-and-out noble character more freely.

\subsection{The Importance of "Jumping" in Chinese Classical Dance "The Soul of the Terracotta Warriors"}

In classical dance works, there are a lot of jumping movements in "The Soul of the Terracotta Warriors". The jumping movement runs through the entire dance work of "The Soul of the Terracotta Warriors", which played a certain role in expressing the emotions of the dancers [3]. In the dance, a large number of jumping movements change with the deepening of the dance plot, which drives the emotions of the audience. In the first half of the dance, the dancer plays the role of Terracotta Warriors. After being put on a heavy armor to break free, the dancer's frontal split jump is full of anger. On the one hand, it shows the pain and unwillingness in the Terracotta Warriors' hearts for being restrained, and the struggle, on the other hand, also brings the audience a great spirit of resistance, which embodies the unyielding of the Terracotta Warriors to be oppressed and their desire for freedom. In the middle of the dance, the Terracotta Warriors' heroic spirit of fighting the enemy courageously was demonstrated. The dancer's jumping movements were enthusiastic and full of passion, creating a high-spirited national hero role for the audience. The audience can better feel the progress of the dance plot and the connotation of the work. Throughout the work, the dancers use a lot of jumping movements to convey the emotion of the work to the audience. In addition, in the process of dance performance, if there is no jumping movement and emotional injection, it will give people a dull and ordinary feeling like chewing wax. Without the emotional interaction brought about by the jumping action, the work of "The Soul of the Terracotta Warriors" will also lose its soul, and it would be really just a sculpture that lost the pursuit of freedom, the desire for freedom and the 
demeanor of defending the country. Jumping is the sender of emotions. All emotions are expressed on the premise of jumping. Emotions can be accurately and profoundly expressed whether they are happy, melancholic, or angry [4]. Injecting emotions with jumping movements, the combination of movements and emotions allows the audience to feel the emotions the dancers want to express.

\section{CONCLUSION}

After all, dance jumping training is for dancers and dance works. In the dance performances, dancers learn to dance jumping movements, rather than simply learning dance jumping skills, so that they can better present the jumping training effect to the audience. Jumping training is very beneficial to the improvement of dancers' physical fitness and ability. Through jumping training, actors can bring better visual enjoyment to the audience, which is also of great help to prolong the life of the dancers on stage.

\section{AUTHORS' CONTRIBUTIONS}

This paper is independently completed by Yanchi Zhou.

\section{REFERENCES}

[1] Shen Yuanmin, Basic Skill Training Teaching Method of Chinese Classical Dance [M]. Shanghai Music Publishing House, 2004 (09). (in Chinese)

[2] Zeng Yue, Analysis of the Content and Function of Big Jump in the Basic Skills of Chinese Classical Dance [J]. Trade Union's Tribune, 2013 (03). (in Chinese)

[3] Wang Haiying, About The Training Principle and Method of Dance Jumping Skill [J]. Studies In National Art, 2001 (04). (in Chinese)

[4] Liu Xiahong, On the Emotional Expression of Chinese Classical Dance Through "The Soul of the Terracotta Warriors" [J]. Journal of Xingtai University, 2013 (03). (in Chinese) 